\title{
Bibliography and references
}

Bourdon, Jérome. 2004. 'Live Television is still Alive', in Allen, Robert Clyde and Hill, Annette (eds), The Television Studies Reader, Routledge

Fletcher, J., Kirby, D.G. and Cunningham, S. 2006. BBC Research White Paper WHP 141, Tapeless and Paperless: Automating Workflow in TV Studio Production, BBC, September

Fraser, Cathie. 1990. The Production Assistant's Survival Guide, BBC Television Training.

Grau, O., Pullen, T. and Thomas, G.A. 2004. BBC R \& D White Paper WHP 086, A Combined Studio Production System for 3D Capturing of Live Action and Immersive Actor Feedback, BBC, April

MacDonald, M. (ed.) 1972. Chambers Twentieth Century Dictionary, W \& R Chambers Ltd

McKee, Robert. 1992. Story: Substance, Structure, Style and the Principles of Screenwriting, Methuen

Millerson, Gerald. 1999. Lighting for TV and Film, Focal Press

Owens, Jim. 2006. Television Sports Production, Focal Press

Phillips, Brian. 1987. Stand by Studio!, BBC Television Training

Rowlands, Avril. 1993. The Television PA's Handbook, Focal Press

Singleton-Turner, Roger and Partridge, Gill. 1988. Continuity Notes, BBC Television Training

Singleton-Turner, Roger. 1994. Television and Children, 1994 BBC Television Training, Borehamwood)

Singleton-Turner, Roger. 1999. Children Acting on Television, A \& C Black

Truss, Lynne. 2003. Eats, Shoots \& Leaves, Profile Books Ltd

University of Sunderland School of Arts, Media Design and Cultural Studies. n.d. Television Studio Operations - Health and Safety in the Studio, Crew Roles and Responsibilities, document for internal circulation only

Ward, Peter. 2001. Studio and Outside Broadcast Camerawork: A Guide to MultiCamerawork Production, Focal Press 
Ward, Peter. 2003. Picture Composition for Film and Television, Elsevier

Watts, Harris. 1997. On Camera (revised edition), AAVO

Webber, R. (ed.) 2001. Walmington Goes to War, Orion Books

\section{Fiction}

Adams, Douglas. 2003. The Salmon of Doubt (posthumously edited collection of writings), Pan/Macmillan

Carroll, Lewis. 1865. Alice's Adventures in Wonderland, Macmillan

Eliot, T.S. 1939. Old Possum's Book of Practical Cats, Faber

Fielding, Henry. 1749. Tom Jones, A. Millar, over against Catherine Street in the Strand

Pratchett, Terry. 1998. The Last Continent, Corgi Books

Wilde, Oscar. 1895. The Importance of Being Earnest, Smithers and Co.

\section{Periodicals}

\section{Broadcast, London, Emap Communications}

7 March 2008, Pennington, Adrian, Perfectionist at Work - Costume Design, p.26

23 May 2008, Carter, Meg, Soundtrack Selection, pp.28-9

8 August 2008, Pennington, Adrian, Format Frenzy, p.22

14 November 2008, Pennington, Adrian, Digilab Launches New Tapeless Shoot Service, p.11

21 November 2008, Pennington Adrian and Parker, Robin, The 360-Degree Turnaround, p.21

19 December 2008, Brittain, Nicola, BSkyB Airs Stereo 3D Demos, p.10

27 February 2009, Sherry, Tom, Analysis: The Dawn of Discount Drama, p.19

6 March 2009, Curtis, Chris, Filling in the Details of a Blank Canvas, p. 15

22 May 2009, Pennington, Adrian, Getting under the Skin of HD Strategy, p.16

29 May 2009, Pennington, Adrian, Seeking Professional Help, p.36

5 June 2009, Pennington, Adrian, Top 10 Tips for Producing Cheaper TV, p.20

24 July 2009, Pennington, Adrian, Filming another Dimension, p.33

21 August 2009, Strauss, W., Sky1 Orders Twofour to Shoot Noel in 3D, p.16

4 September 2009, Technology Focus For-A VRCAM, p.17

22 January 2010, Stout, Andy, Sound of Things to Come/The Future for 5.1, pp.32-3

26 March 2010, Pennington, Adrian, Making the Technology Work, p.19

26 March 2010, Pennington, Adrian Managing 3D Made Easy, p.25

6 August 2010, Wood, David, Tapeless Workflow: The Lessons Learned, p.24

25 February 2011, Bevir, George, 3ality cuts cost of 3D shooting, p.16 
Stage, Screen and Radio the journal of the Broadcasting, Entertainment and Theatre Union (BECTU): June 2008, p. 14.

\section{Internet sources}

Most of these sites were accessed between January 2008 and December 2009.

ActorsLife.com, Director: Scott Cummins, Los Angeles theatre, film director/actor: www.actorslife.com/article.php?id=204

allmovie (All Media Guide), Glossary: Invisible Cutting: www.allmovie.com/glossary/term/invisible+cutting

Baird Television: www.bairdtelevision.com

BBC Historic Figures John Logie Baird: www.bbc.co.uk/history/historic_figures/baird_logie.shtml

BBC Production Automation 2005-06: www.bbc.co.uk/rd/pubs/annual-review/rev_06/bbcrdar06-creative.pdf

BBC Research automated tapeless production: www.bbc.co.uk/rd/projects/tapeless-production/indexp2.shtml

BBC Research, Music Production Planning: www.bbc.co.uk/rd/projects/music-production-planning/new-approach.shtml

BBC Research, Music Production Planning, Creating the Script: www.bbc.co.uk/rd/projects/music-production-planning/the-script.shtml

BBC Southampton: www.bbc.co.uk/southampton/music.ogwtindex.shtml

bizhelp Critical Path Analysis: www.bizhelp24.com/small-business/critical-path-analysis.html

Brett, Jeremy: www.jeremybrett.info/jb_joan_wgbh.html

Citizens Advice (National Association of Citizens Advice Bureaux), Employment in England: Young People and Employment 2002-08: www.adviceguide.org. uk/inex/life/employment/young_people_and_employment.htm

Cole, Matthew A Tedious Explanation of the f/stop 2003/10: www.uscoles.co/fstop.htm

Core Sound CoreSound TetraMic: www.core-sound.com/TetraMic/1.php

The CyberCollege-InternetCampus Makeup for TV and Film 7 March 2008: www.cybercollege.com/makeup.htm 
Dooley, Wes PDF by Ron Streicher The DeccaTree - It's not just for Stereo any more, originally published in Mix Magazine 2003: www.wesdooley.com/pdf/Surround_Sound_Decca_Tree-urtext.pdf

eHow What is LTO Tape? Keith Allen. June 2010: www.ehow.com/about_6646965_lto-tape_html

Film of the Year 1914 The Rise and Fall of Italy's Silent Spectacles: http://filmyear.typepad.com/blog/2006/10/decline_and_fal.html

The First British Television Play: www.tvdawn.com/mwfihm

The Free Dictionary: http://encyclopedia2.thefreedictionary.com/Companded

Holophone Holophone H2 Pro: www.holophone.com/technical.html

Hosgood, Steve All You Ever Wanted to Know About NICAM but Were Afraid to Ask, 1995, 1996, 1997: http://tallyho.bc.nu/ steve/nicam.html

HowStuffWorks Harris, Tom, 'How Cameras Work', 21 March 2001: http://electronics.howstuffworks.com/camera.htm

HowStuffWorks Freeman, Shanna, 'How Muppets Work', 18 September 2007: http://entertainment.howstuffworks.com/muppet.htm

IMDb: www.imdb.com/title/tt0020402

ITV Technical Requirements: www.itv.com/documents/pdf/Technical\%Requirem ents\%for\%Programme\%Material\%20Commissioned\%20by\%20ITV.pdf (August 2007)

Jackson, Blair: mixonline.com/mag/audio_foley_recording/index.html (September 2005)

Karsten Gerloff, UNU-MERIT, Low-Cost High Tech: BBC Tries out Open Source-Based Tapeless Recording: http://ec.europa.eu/idabc/en/ document/7411

Kempton, Martin, An Incomplete History of London's Television Studios: www.tvstudiohistory.co.uk (2006)

Media College.com Pickup Shots: www.mediacollege.com/video/shots/pickup.html

Media College.com HMI Lights: www.mediacollege.com/lighting/types/hmi.html

Media College.com How Do Microphones Work?: www.mediacollege.com/ audio/microphones/how-microphones-work.html

Merriam-Webster OnLine Search:

www.merriam-webster.com/dictionary/incidental+music 


\section{2}

Mind Tools Critical Path Analysis and PERT Charts: www.mindtools.com/critpath.html.

The Museum of Broadcast Communications, Jacobs, Jason, Studio One US Anthology Drama: www.museum.tv/archives/etv/S/htmlS/studioone/studioone.htm

Panasonic, Full HD 3DTV: www.panasonic.com/3D

Peters, Jaquelyn, Brecht and Alienation: http://courses.essex.ac.uk/LT/LT204/brechtnotes.htm

Rittermann, M. and Schuldt, M., 3D Television Production Based on MPEG-4 Principles: http://wscg.zcu.cz/wscg2003/Papers_2003/D11.pdf

Seel, R., Welcome to Richard Seel's Writings: www.articles.adsoft.org/postproduction (2005)

TalkTalk Encyclopedia displacement activity http://www.talktalk.co.uk/reference/encyclopaedia/hutchinson/m0007993. html

TV.com Softly, Softly: www.tv.com/softly-softly/show/8544/summary.html

VideoUniversity.com: www.videouniversity.com/articles/ adjusting-the-back-focus-of-a-lens

Wikipedia, 180 Degree Rule: http://en.wikipedia.org/wiki/180degreerule

Wikipedia, 3D Display: http://en.wikipedia.org/wiki/3D_display

Wikipedia, Aspect Ratio (Image):

http://en.wikipedia.org/wiki/Aspect_ratio_(image)

Wikipedia, Charades: http://en.wikipedia.org/wiki/Charades

Wikipedia, Dorothy Fields: http://en.wikipedia.org/wiki/Dorothy_Fields

Wikipedia, Shadow Play: http://en.wikipedia.org/wiki/Shadow_play

Wikipedia, So You Think You Can Dance: http://en.wikipedia.org/wiki/So_You_Think_You_Can_Dance

Wikipedia, Sooty: http://en.wikipedia.org/wiki/Sooty

Wikipedia, Super Hi-Vision: http://en.wikipedia.org/wiki/Super_Hi-Vision

Wikipedia, Top of the Pops: http://en.wikipedia.org/wiki/Top_of_the_Pops

YouTube, The Man with the Flower in his Mouth: www.youtube.com/watch?v=RJoYskwKxsM 